\title{
Corrected Formulation for Spurr Low Viscosity Embedding Medium Using the Replacement Epoxide ERL 4221
}

\author{
E. Ann Ellis
}

Microscopy and Imaging Center, BSBW 119/MS 2257, Texas A\&M University, College Station, TX 77843-2257

The Spurr low viscosity embedding medium [1] used vinyl cyclohexene dioxide (VCD or ERL 4206) as the low viscosity epoxy resin together with nonenyl succinic anhydride (NSA), the flexibilizer DER 736 and the accelerator dimethylaminoethanol (DMAE or S-1). VCD, a cycloaliphatic diepoxide with an average WPE (weight per epoxide equivalent) of 76 was removed from the market in 2005 due to its toxicity and replaced with ERL 4221, a cyclic epoxide with a WPE of 137. Microscopists have been instructed to use ERL 4221 as a direct replacement in the same amounts for VCD in the Spurr formulation. Using ERL 4221 at the same rate as VCD results in a harder, more brittle block which lacks the sectioning qualities of the original formulation. Modifications to the dehydration schedule and reductions in the amount of DMAE have been suggested to improve the quality of the blocks. However, the suggested modifications do not address the real problem with the replacement epoxide. The source of the problem is raising the anhydride:epoxide ratio from $0.88: 1$ in the original formulation to $1.6: 1$ in the formulation by directly substituting ERL 4221. The significance of maintaining an appropriate anhydride:epoxide ratio for a given embedding formulation [2] has not been appreciated in recent years; however, substituting ERL 4221 for VCD reiterates this importance. The corrected formulation presented here maintains the anhydride:epoxide ratio at 0.88:1 [1] and preserves the good sectioning qualities and beam stability of the original Spurr formulation.

Viscosity of the original, standard formulation was 60 centipoise. With the increased WPE of the replacement epoxide there should be an increase in viscosity of the corrected formulation. Viscosity of the new, corrected formulation was determined by comparing flow rates to those of the standard Spurr formulation with VCD. There was a three fold increase in flow rate which gives an approximate viscosity of 180 centipoise for the corrected formulation using ERL 4221. Due to the increased viscosity of ERL 4221, care should be taken to mix the resin components thoroughly. This increased viscosity is still much lower than the viscosity of Epon-Araldite formulations and offers the good cutting qualities and beam stability which many people prefer as compared to formulations based on Luft's Epon [3].

Table 1 shows the corrected standard formulation in grams of Spurr low viscosity embedding media based on batches of 10 grams without the flexibilizer DER 736. Modifications of the formulation for harder or softer blocks are given in Table 2. As in the original formulation, hardness is controlled by varying the amount of the flexibilizer, DER 736, with approximately the same proportions as recommended by Spurr [1]. It is suggested that individuals prepare small batches of resin and vary the amount of DER 736 to determine the optimal amount of flexibilizer for their particular specimens.

Although ERL 4221 (Fig. 1) has a higher molecular weight and viscosity and should not be as toxic as VCD (Fig 2), it has the same parent structure as VCD. The same safety precautions should be 
exercised in handling ERL 4221 and formulations using this epoxide as have been recommended in the past for VCD and the original Spurr formulation [4]. In addition, precautions should be taken to keep resin components and mixtures dry by use of oven dried containers, utensils and embedding molds.

References

[1] A. R. Spurr, J. Ultrastruct. Res. 26(1969)31.

[2] C. W. Geiselman and C. N. Burke, J. Ultrastruct. Res. 43(1973)220.

[3] J. H. Luft, J. Biophys. Biochem. 9(1961)409.

[4] E. A. Ellis, Microscopy Today 11:2 (2003)46.

[5] Aldrich Handbook of Chemicals and Laboratory Equipment. 2000-2001.

[6] A. M. Glauert and P. R. Lewis, Biological Specimen Preparation for Transmission Electron Microscopy, Princeton University Press, Princeton, NJ, 1998.

[7] Appreciation is expressed to John Arnott, Ladd Research, Williston, VT, for providing information on the WPE of ERL 4221 before such information was available to the general public.

TABLE 1: Standard Formulation in Grams

\begin{tabular}{ccccc}
\hline GRAMS & ERL 4221 & NSA & DER 736 & DMAE \\
\hline 10 & 4.10 & 5.90 & 1.43 & 0.1 \\
20 & 8.20 & 11.80 & 2.86 & 0.2 \\
30 & 12.30 & 17.70 & 4.29 & 0.3 \\
40 & 16.40 & 23.60 & 5.72 & 0.4 \\
50 & 20.50 & 29.50 & 7.15 & 0.5 \\
\hline
\end{tabular}

TABLE 2: Hard and Soft Formulations in Grams

\begin{tabular}{ccccc}
\hline FORMULATION & ERL 4221 & NSA & DER 736 & DMAE \\
\hline Hard & 4.10 & 5.90 & 0.95 & 0.1 \\
Soft & 4.10 & 5.90 & 1.90 & 0.1 \\
\hline
\end{tabular}

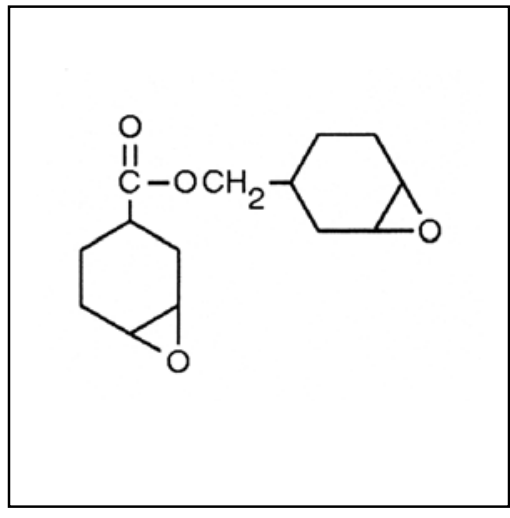

Fig. 1 Structure of ERL 4221 [5].

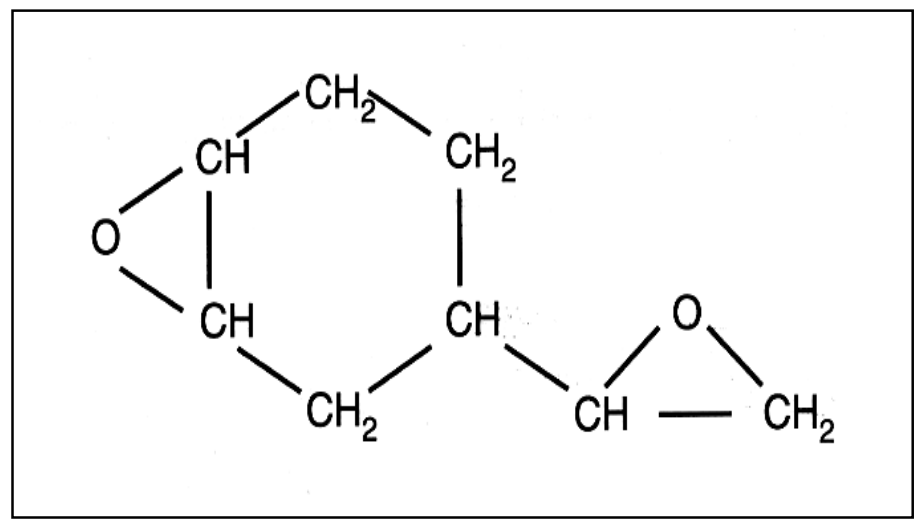

Fig. 2 Structure of VCD [6]. 